\title{
The Relationship between Levels of Pain, Anxiety and Depression with the Health-Related Quality of Life among Chronic Low Back Pain Patients
}

\author{
Zaid Ehsan Suliman Alkhwaldeh \\ Master Student \\ Dr. Yousef Abd Al Wahab Abuhmaidan \\ Professor \\ University of Jordan \\ Applied Behavioral Psychology \\ University of Jordan
}

\begin{abstract}
The present study aimed to investigate the relationship between levels of pain, anxiety and depression with the healthrelated quality of life among patients with chronic low back pain seeking treatment in some physiotherapy centers. Sample consisted of (216) patients at the Royal Rehabilitation Centre at Al-Husain Medical City Hospital and the Department of Physical Therapy at Prince Hashem Bin Al Hussein Military Hospital. To measure the variables of the study, we used of the following tools: Pain Visual Analog Scale (VAS), State-Trait Anxiety Inventory (STAI), and Beck Depression Inventory (BDI). The Health-related quality of life measured by 36-item Short Form Health Survey (SF36). All tools characterized by appropriate degree of validity and reliability. After conducting statistical analysis, the study concluded that there was severely degree of decline in the overall measure of the health-related quality in the overall eight areas of the health-related quality of life scale with a mean of (48.63).
\end{abstract}

The results also showed that there was a noticeable decline in very severe degree in the field of the limited role as a result of physical health and in the field of the limited role as a result of emotional problems. Additionally, the results showed severe decline in health-related quality of life in the other fields of health-related quality of life (bodily functions, energy and fatigue, emotional well-being, social function, bodily pain, general health). The range averages for the fields of health-related quality of life were between (35.49-59.05) where the highest mean was for field of physical functions (59.05) and the lowest mean was for the field of limited role as a result physical health (35.49).

The study showed a significant positive correlation between pain and depression, as well as presence of a significant positive correlation between pain and anxiety. Also it showed that a significant negative correlation between the level of health-related quality of life, and all study variables (pain, anxiety, depression).

The results indicated that anxiety alone was able to explain a percentage of (29.7\%) of health-related quality of life and was the highest explanatory rate, and that pain alone managed to explain a percentage of (11.5\%), while the explanatory of depression ability was (4.5\%) and the total of what could be interpreted from the variables (anxiety, pain, and depression) was (45.7\%) of the total variation in the Health-Related quality of healthy life. The study also showed that although the presence of correlation between the health-related quality of life and other variables on sex with both males and females as well as the presence of correlation between the health-related quality of life and other variables (anxiety, pain, and depression) and the level of education (secondary education or less and those with university degree), there was no statistically significant difference between the health-related quality of life and other variables (pain, anxiety, and depression) and sex or different level of education.

In conclusion, this study recommends conducting more studies about psychology of pain in people with low back pain. Having more studies about psychology of pain in this population would improve the understanding of Physicians who are concerned and physical therapists to this important issue. Consequently, better understanding would promote prevention, diagnosis and appropriate management especially for people with chronic low back pain.

Keywords: pain, anxiety, depression, quality of life

\section{Introduction}

The problem of pain is a great burden on societies and nations; about one-third of the population of the United States suffered chronic pain in their lives, the American community endures a large cost ranging between (500-600) billion dollars annually, this cost far exceeds the total cost associated with other diseases such as diabetes, heart disease, and cancer. Despite these exorbitant costs, chronic pain is deficient (Pizzo PA, Clark NM (2012), diseases, disorders and 
pain syndromes vary, but are similar to requiring therapeutic intervention by a multidisciplinary team; such as drug therapy, behavioral psychological interventions, and qualitative interventions (Sirianni J, Ibrahim M, Patwardhan A (2015). In an international survey of 18 countries, a sample of 42,249 respondents, which was conducted over a period of 12 months; it was found that the prevalence of chronic pain was about (37\%) in developed countries, with a percentage of (41\%) in developing countries (Tsang et al., 2008). According to estimates of this phenomenon in a 2011 report by the Institute of Medicine, 116 million adults in the United States have different forms of chronic pain, common among children and adolescents. The proportion of children with chronic pain is about 25\% (Perquin et al. 2000). These data show that the total of chronic pain sufferers is more prevalent than the total of those suffering from heart disease, diabetes and cancer. Chronic pain also has a negative impact on health, quality of life, negative correlation with mood, physical dysfunction and contributes to depression and low levels of activity (Berg-Emons et al. 2007). Acute Pain is a physiological response that warns the body of a defect, while chronic pain is caused by a pathological condition that is related to different diseases or a particular disease.

Pain is one of the important and complex phenomena and the problems and symptoms common in many patients and of different ages, there are many studies and models and theories that tried to explain the phenomenon of pain to contribute to the assessment and treatment of cases better. Over the past few decades, chronic diseases have increased markedly as part of an increase in the relative size of the disease. Dealing with pain and understanding its psychological, physical, social, and emotional dimensions is of great importance to achieve higher awareness of specialists and physicians, and to help patients with chronic pain and their families overcome the emotional and social effects of the disease and treat it. Chronic complaints of chronic low back pain include chronic anxiety and depression. Thus, work efforts have begun to focus on activating psychological care with the onset of primary medical care, training of health professionals, including physiotherapists on psychological methods of assessment and even therapeutic interventions to help patients with chronic pain (Lindsay, Paul, 2000). Health means not only the absence of disability, but also the state of physical, mental and social completeness (WHO, 1946).

Lower back pain is a major public health problem that has a direct and indirect relationship with cost and causes disabling disease and limits the quality of life. Psychological factors may have a greater impact on disability and quality of life than the pain itself.

The World Health Organization (WHO, 2003) considers low back pain to be the leading cause of quality of life (QOL), low performance, and leads to disability. Lower back pain is the most common. The majority of the cases are due to non-specific causes. Back pain is the most common and usually disappears automatically. It does not last more than 3 months. Psychological problems arise in the chronic stage.

\section{Problem of the Study}

The researcher noted through his work in the field of clinical training for physical therapy students in physiotherapy units of a number of government and military hospitals in the Hashemite Kingdom of Jordan. That patients with chronic low back pain show many symptoms of low quality of life, such as feelings of distress and anxiety and low mood, and complain of low performance and normal physical activities, their health and psychological condition interferes with social activities. Since neurologists, orthopedic, orthopedic and physiotherapists focus on the diagnosis and evaluation of low back pain patients through clinical examination and medical imaging of all kinds. In addition to patient observation and examination by evaluating the severity of pain, muscle strength, and specialized clinical tests. They neglect to assess each of the psychological factors that accompany the pain, and assess the quality of life for patients. According to studies indicating that pain is associated with the feeling of many patients with low back pain down the quality of healthy life, and what accompanies the pain of feeling anxious and depressed. This study is intended to determine the effect of low back pain on the mental state of these patients and on the quality of their healthy lives.

As well as to identify the relationship of pain and associated symptoms of anxiety, depression and quality of life in patients with chronic low back pain, who review clinics, physiotherapy and rehabilitation.

Questions of the Study

The study attempts to answer the following questions:

1. What is the quality of life in patients with chronic low back pain?

2. Is there a relationship between the quality of healthy life and the level of pain in patients with chronic low back pain?

3. Is there a relationship between the quality of life and the level of anxiety in patients with chronic low back pain? 
4. Is there a relationship between healthy quality of life and level of depression in patients with chronic low back pain?

5. Does the relationship between quality of life and other variables (pain, anxiety, and depression) vary depending on gender?

6. Does the relationship between the quality of healthy life and the other variables (pain, anxiety, and depression) vary according to the level of education?

7. To what extent does the level of pain, anxiety, and depression explain the quality of healthy life in patients with chronic low back pain?

8. Is there a relationship between the level of pain and the level of depression?

9. Is there a relationship between pain level and anxiety level?

\section{Purpose of the study}

The aim of this study is to explore the relationship between the quality of life, health, pain and accompanying symptoms of anxiety and depression in patients with chronic low back pain.

\section{The importance of the study}

The importance of this study appears in providing psychological enrichment for doctors, Physiotherapists, and workers in various medical and psychological fields related to the care of patients with chronic low back pain, for a deeper understanding of the psychological, social and vital dimensions of pain and associated symptoms of anxiety and depression. And the extent to which these patients are affected or reduced. Thus contributing to greater knowledge about the effectiveness of clinical and therapeutic interventions provided by clinicians

\section{The limits of the study}

The current study is limited to patients with chronic low back pain who visit physiotherapy centers.

\section{Study determinants}

The study was limited to a sample of patients with chronic low back pain who were suffering from pain for 3 months and above. They were undergoing physical therapy programs at the Royal Rehabilitation Center in Al Hassan Medical City and the Physiotherapy Department at Prince Hashim Bin Al Hussein Military Hospital.

\section{Previous Studies}

Jarrar (2011) conducted a study aimed at identifying the extent to which pain and depression contributed to the increased feeling of disability in patients with low back pain who are visiting clinics, hospitals and physiotherapy centers in Amman, where the sample consisted of (123) patients who completed the Beck Depression Scale version II, the pain-related disability index and the pain score scale. The results showed an increase in levels of pain, depression and feeling of disability. A statistically significant association was found between the level of pain and the level of feeling of disability and the level of depression and the level of feeling disabled, the study also showed that depression explains the feeling of disability more than the pain itself. Alshami (2014) conducted a study in Saudi Arabia aimed at investigating the physical and psychological aspects of low back pain patients who visit King Fahad University Hospital in Al Khobar, (31) patients with Depression, Anxiety and Stress Scale (DASS), and the Visual Analogue Scale (VAS), in addition to the International Physical Activity Questionnaire (IPAQ), the sample was selected from those patients who complained of pain for more than one year. Their pain was greater than degree (3), who took medication to relieve the pain, and the pain reduced their physical activity. As for the control group, it consisted of (30) people whose pain was less than (3) throughout their lives and did not have to take sedatives or to reduce their physical activity, the study found that patients with chronic low back pain had a higher stress level than the control group, the researcher recommended further studies and focus on the psychological side associated with low back pain along with the physical side, and the need for evaluation and psychological treatment necessary for patients.

There have been many studies on quality of life such as Al-Dawood (2007), conducted in Saudi Arabia to assess the quality of life in patients with diabetes to see the specific indicators of the disease that affect daily activities from their point of view. The researcher used the descriptive method of study through the cross-sectional sample, the study used a health survey model (BRAND-36) which measures quality of life through (8) dimensions, the sample consisted of (154) participants randomly selected from the center of Prince Salman bin Abdul Aziz located in the Kingdom of Saudi Arabia in the capital Riyadh, the study concluded that diabetes has a clear effect on people with diabetes.

The results showed a clear decline in quality of life, especially that diabetes is a chronic disease for life and requires constant attention and attention to measure the level of glucose in the blood, diet, and commitment to medication, were low quality of life included many dimensions such as low physical, emotional and social functions and low energy. Hong et al. (2014) conducted a study in Korea recently published to assess anxiety, depression, sleep problems and 
healthy quality of life in patients with low back pain, the sample consisted of 47 patients with chronic lower back pain for 3 months or more. The control group consisted of 47 healthy people, who matched the gender and age of the patient sample. Both groups were evaluated by their response to the Beck Depression List, the Beck Diagnostic Test, and the Oswestry Disability Index (ODI), and the Pittsburgh Sleep Quality Index (PSQI), and the Short-Health Health Survey (SF-36). The results of the study showed that there was an increase in the levels of anxiety, disability and depression in the sample of patients compared to the control group, in addition to a clear decrease in the quality of life of health patients compared to the control sample, the incidence of depression in the patient sample was much higher than the control sample, but there was no difference in the quality of sleep between the two groups. The study concluded that patients with chronic lower back pain exhibit a marked functional disability, and a significant decline in mental state, and a marked decline in the quality of healthy life. The study recommended that patients be evaluated in order to provide appropriate psychological care.

In a study by Baena-Beato et al. (2014), the aim was to identify the association between pain, disability, quality of life, and cognitive behavioral factors in patients with low back pain. The purpose of this study was to examine the correlations between the outcomes of variables such as pain severity, disability, and quality of life health and to show these findings and the psychological risk factors for chronic low back pain such as depression, characteristic anxiety, avoidance, and the tolerance associated with pain responses at two different points in the assessment. Initially, 52 patients with chronic low back pain were examined; after 6 months of treatment, the two-variable relationships between pain severity, disability, quality of life and the psychological variables were examined at the present time. In addition, differences between links at the baseline and follow-up were examined. Significant differences were found between correlations, with low baseline links and high correlation at follow-up. The study also showed a positive correlation to healthy quality of life with related endurance, it is important to highlight assessment time points that play an important role in lower back pain.

Another study by (Adorno \& Brasil-Neto, 2013) aimed at measuring the quality of healthy life in a sample of patients with chronic nonspecific low back pain. The sample consisted of (30) patients randomly divided into three groups according to the treatment method: ISO group therapy group ISO stretching. The second group used GCR therapy (Global Postural Reeducation). The third group used the first and second techniques with the groups together, the ISO + GPR group. The patients were evaluated for natural spine treatment, the Visual Analog Scale of Pain and the SF-36 short form of health during a packaged questionnaire, the first evaluation was performed before the first session, the second evaluation after 3 months of treatment sessions, and the final evaluation where the reassessment was reassessed two months after the second evaluation. The study concluded that the use of the integrated method (ISO + GPR) for physiotherapy resulted in significantly reducing the pain level and qualitative at ( $p<0.001)$. The quality of healthy life improved after intervention on the GPR group at $\mathrm{p}<0.001$. The study concluded that ISO treatment was more effective in the final evaluation phase.

\section{Method and procedures}

\section{Population of the Study}

The study population consists of patients with chronic low back pain, who visit orthopedic clinics and physiotherapy and rehabilitation departments at the Royal Rehabilitation Center in Al Hussein Medical City and Prince Hashem bin Al Hussein Military Hospital.

\section{Sample of the Study}

A sample of patients with chronic low back pain, aged 18 years and older, and both sexes, who are visiting the Physical Therapy and Rehabilitation Department at Royal Medical Services Hospitals. The sample consisted of patients who visited the physiotherapy department for a period of 3 months between January and March of those diagnosed with chronic and non-specific chronic low back pain. The number of patients with chronic low back pain reached (216) patients.

\section{Methodology of the study}

This study adopted the descriptive approach to suit the study variables.

\section{Study variables}

- Pain level in patients with low back pain.

- The level of anxiety in patients with low back pain.

- The level of depression in patients with low back pain.

- Quality of life in patients with low back pain. 
Table (1): Distribution of Study Sample Individuals by Age, Gender and Educational Level Variables

\begin{tabular}{lll}
\hline \multicolumn{1}{c}{ Age } & Frequency & Percentage\% \\
\hline From 18 to 20 years old & 3 & 1.4 \\
From 21 to 26 years & 27 & 12.5 \\
From 27 to 32 years old & 50 & 23.1 \\
From 33 years and older & 136 & 63.0 \\
Total & 216 & 100.0 \\
Gender & Frequency & Percentage\% \\
Male & 93 & 43.05 \\
Female & 123 & 56.94 \\
Total & 216 & 100.0 \\
Educational level & Frequency & Percentage\% \\
Illiterate & 6 & 2.8 \\
Primary & 10 & 4.6 \\
Preparatory & 23 & 10.6 \\
Secondary & 48 & 22.2 \\
Undergraduate studies & 129 & 59.7 \\
Total & 216 & 100.0 \\
\hline
\end{tabular}

From Table (1) we note that (1.4\%) of the sample of the study is between the ages of (15 to 20) years, and that (12.5\%) of the sample of the study are aged between twenty-one and twenty-six years, and $23.1 \%$ of the sample of the study were between the ages of twenty-seven and thirty-two years, and $63.0 \%$ of the sample of the sample were aged thirtythree years and over, from the above table, we see that the largest group of individuals in the study sample were individuals aged over thirty-three. We also note that (43.05\%) of the study sample was male, and (56.94\%) of the study sample were females. It was also found that $2.8 \%$ of the sample members of the study are illiterate individuals who do not know how to read or write, and that $4.6 \%$ of the study sample are individuals who have completed the primary stage of education, and that $10.6 \%$ of the sample of the study completed the preparatory stage of education, and $22.2 \%$ of the sample of the study completed the secondary stage of education, and $59.7 \%$ of the sample of the study were individuals who completed their university studies, and from above we note that the highest category of sample members of the study consisted of individuals who had completed their undergraduate studies.

\section{Study Tools}

The researcher used the following tools in his study:

- (SF-36) short form healthy survey.

- Visual Analog Scale for Pain (VAS Pain).

- Beck Depression Inventory (BDI)

- State-Trait Anxiety Inventory (STAI)

\section{Quality of healthy life Scale}

The scale consists of (36) items divided into (8) domains, ranging from 0-100 in all health quality standards in each area of quality of life, where (zero) is the worst case of quality of healthy life, while degree 100 means the best quality of life.

\section{Validity and Reliability of the scale}

Al-Hourani conducted a study on the quality of life in northern Jordan through the use of a short-health survey (SF-36), which includes eight dimensions: physical function, Functional role/ physical role/ emotional, energy / fatigue, wellbeing, social functioning, pain, general health, health change. This scale includes (36) paragraphs covering these dimensions. This study adopted this scale after deriving from the Arabic-Lebanese version. Based on the above, the study concluded that the quality of healthy life scale is reliable and valid among the Jordanian population.

\section{Reliability of the scale}

In the current study according to the coefficient of internal consistency as an indicator of the reliability of the test (0.89) which is a suitable coefficient and fulfills the purposes of the current study. The quality of life in the current study sample was calculated by setting a default score of ( 0 to 100), with 100 indicating the best quality of life and (0) indicating the worst quality of life. 


\section{Pain Visual Analog Scale}

It is a simple tool designed to measure the intensity of the pain so that the patient refers to the degree that expresses his awareness of the severity of the pain on a virtual scale of (0-10), where (zero) means the absence of pain and (10) means the highest severity of pain imaginable. In the current study, the level of pain was calculated through a mean average so that the degree of (1-3) means mild pain, and the degree between (4-6) mean moderate pain, the degree ranging from (7-10) means severe pain.

\section{Testing the Status and anxiety trait}

This test was prepared by Spielberger et al in 1983. The test measures anxiety as a case and characterization. It includes two sub-tests of anxiety measurement as a feature that measures general anxiety and anxiety as a case that measures the current anxiety level of the individual. Each sub-test consists of (20) paragraphs. the study use anxiety test as the only case where it measures feelings of tension, fear, nervousness, and discomfort at the present moment.

The total score of the scale ranges between (20) and (80) score is calculated after the selection of answers by the examinee and there are four degrees ranging from (1) to (4).

\section{Validity and Reliability of the test}

In the current study according to the coefficient of internal consistency as an indicator of the stability of the test at (0.90) which is a suitable coefficient and fulfills the purposes of the current study.

\section{Study procedures}

In order to achieve the objectives of the study, the procedures were carried out according to the following steps:

- Verify the validity and reliability of study tools.

- Determine the number of members of the study community by referring to official sources in the Royal Medical Services.

- Application of study tools to a sample of individuals diagnosed with chronic low back pain.

- (350) questionnaires were distributed for two months, 250 were retrieved. The researcher excluded (34) because of a lack of questionnaire data. The total number of valid questionnaires for the study was (216) questionnaires.

- Data collection and validation for statistical analysis, and then using of statistical analysis software (SPSS) to analyze data and obtain results.

\section{Results of the study}

Results related to the first question: What is the quality of life in patients with chronic low back pain?

To answer this question, the Average and standard deviations were calculated. Table (2) shows the quality of life in patients with chronic low back pain.

Table (2): Arithmetic averages and standard deviations of the standard of living quality in patients with chronic low back pain

\begin{tabular}{lllll}
\hline Domain & $\begin{array}{l}\text { Arithmetic } \\
\text { average }\end{array}$ & $\begin{array}{l}\text { The degree of } \\
\text { decline in } \\
\text { quality } \\
\text { healthy life }\end{array}$ & $\begin{array}{l}\text { standard } \\
\text { of } \\
\text { deviation }\end{array}$ & Ranking \\
& & Severe & 23.39 & 1 \\
\hline Physical functions & 59.05 & Severe & 24.20 & 2 \\
Social function & 56.31 & Severe & 21.99 & 3 \\
Emotional Wellbeing & 54.06 & Severe & 10.86 & 4 \\
$\begin{array}{l}\text { General Health } \\
\text { Physical pain }\end{array}$ & 51.36 & Severe & 21.01 & 5 \\
$\begin{array}{l}\text { Energy and fatigue } \\
\text { Limited role due to emotional } \\
\text { problems }\end{array}$ & 44.47 & Severe & 19.39 & 6 \\
$\begin{array}{l}\text { Limited role due to physical health } \\
\text { Quality of Healthy Living (total) }\end{array}$ & 37.91 & Very severe & 41.12 & 7 \\
\hline
\end{tabular}

It is clear from Table (2) that the averages for fields of health quality of life ranged between (35.49 - 59.05) and that the highest average arithmetic it was the field of Physical functions in which its arithmetic average was (59.05) by standard deviation (23.39), and that the lowest average arithmetic was the area of limited role due to physical health, with an average of (35.49) with a standard deviation of (34.99). And the arithmetic average of the quality of healthy life (Total) in all areas (48.63) with a standard deviation (15.47), which means that the quality of life is severely affected. 
Results related to the second question: Is there a relationship between the quality of healthy life and the level of pain in patients with chronic low back pain? To answer this question, the Pearson correlation coefficient was calculated between healthy quality of life and level of pain in patients with chronic low back pain.

Table 3: Pearson correlation coefficient between healthy quality of life and level of pain in patients with chronic low back pain

\begin{tabular}{lll}
\hline Domain & Level of pain & \\
\cline { 2 - 3 } Physical functions & Correlation & Significance \\
Limited role due to physical health & $.283^{*} *_{-}$ & 0.001 \\
Limited role due to emotional problems & $.211^{* *_{-}}$ & 0.001 \\
Energy and fatigue & $.0 .255^{*_{-}}$ & 0.001 \\
Emotional Wellbeing & $.32 *^{*}$ & 0.001 \\
Social function & $.203^{* *_{-}}$ & 0.001 \\
Physical pain & $.263^{* *_{-}}$ & 0.001 \\
General Health & $.549^{* *_{-}}$ & 0.001 \\
\hline
\end{tabular}

From Table (3) it is clear that correlation coefficients between physical function, limited role due to physical health, Limited role due to emotional problems, energy and fatigue, emotional well-being, social function, physical pain, public health, with the level of pain, which amounted to (-0.283،0.211- $0.255-60.324-60.203-60.263-60.549-، 0.217-)$ all of these values are at the level of 0.05 and below, so there is a negative correlation between the quality of life and the level of pain in patients with chronic low back pain.

Results related to the third question: Is there a relationship between the quality of life and the level of anxiety in patients with chronic low back pain? To answer this question, the Pearson correlation coefficient was calculated between healthy quality of life and level of anxiety in patients with chronic low back pain.

Table (4): Pearson correlation coefficient between the level of healthy quality of life and level of anxiety in patients with chronic low back pain

\begin{tabular}{lll}
\hline Domain & Anxiety & \\
\cline { 2 - 3 } & Correlation & Significance \\
Physical functions & $-.213^{* *}$ & 0.001 \\
Limited role due to physical health & $-.205^{* *}$ & 0.001 \\
Limited role due to emotional problems & $-.316^{* *}$ & 0.001 \\
Energy and fatigue & $-.526^{* *}$ & 0.001 \\
Emotional Wellbeing & $-.693^{* *}$ & 0.001 \\
Social function & $-.463^{* *}$ & 0.001 \\
Physical pain & $-.339^{* *}$ & 0.001 \\
General Health & $-.264^{* *}$ & 0.001 \\
\hline
\end{tabular}

Table (4) shows that correlation coefficients are between (Physical functions, limited role due to physical health, limited role due to emotional problems, energy and fatigue, emotional well-being, social function, Physical pain, public health) with the level of anxiety, which amounted to $(-0.213,-0.202,-0.310,-0.526,-0.693-,-0463-, 0.339,-0.264-)$.

Results related to the fourth question: Is there a relationship between quality of healthy life and level of depression in patients with chronic low back pain? To answer this question, the Pearson correlation coefficient was calculated between healthy quality of life and level of depression in patients with chronic low back pain.

Table 5: Pearson correlation coefficient between healthy quality of life and level of depression in patients with chronic low back pain

\begin{tabular}{lll}
\hline Domain & Depression & \\
\cline { 2 - 3 } & Correlation & Significance \\
Physical functions & $-.357^{* *}$ & 0.01 \\
Limited role due to physical health & $-.188^{* *}$ & 0.01 \\
Limited role due to emotional problems & $-.224^{* *}$ & 0.01 \\
Energy and fatigue & $-.387^{* *}$ & 0.01 \\
Emotional Wellbeing & $-.607^{* *}$ & 0.01 \\
Social function & $-.476^{* *}$ & 0.01 \\
Physical pain & $-.325^{* *}$ & 0.01 \\
General Health & $-.211^{* *}$ & 0.01 \\
\hline
\end{tabular}


Table (5) shows that correlation coefficients between (Physical functions, limited role due to physical health, limited role due to emotional problems, energy and fatigue, emotional well-being, social function, physical pain, public health) with depression, which reached the level of $(0.357,0.188,-0.23,0.387,0.607,-0.476,-0.32,-0.211)$ all of these values function at a level of 0.05 , therefore, there is a negative correlation between the quality of life and the level of depression in patients with chronic low back pain.

Results related to the fifth question: Does the relationship between quality of life and other variables (pain, anxiety, and depression) vary depending on gender?

To answer this question, the correlation between quality of healthy life and other variables (pain, anxiety, and depression) was calculated according to gender variable, a test was then conducted to derive the significance of the differences between the correlation coefficients according to the gender variable and Table (6) shows the results.

Table (6): Correlations between quality of healthy life, level of pain, anxiety, and depression according to gender variable.

\begin{tabular}{|c|c|c|c|c|c|c|}
\hline \multirow[t]{3}{*}{ variable } & \multicolumn{4}{|c|}{ Quality of life } & \multirow[t]{3}{*}{$\mathrm{Z}$} & \multirow[t]{3}{*}{ Significance } \\
\hline & \multicolumn{2}{|c|}{ Male $\mathrm{N}=(81)$} & \multicolumn{2}{|c|}{ Females $\mathrm{N}=117$} & & \\
\hline & Link & Significance & Link & Significance & & \\
\hline Pain & $.445 *^{*}$ & .001 & $.444 * *_{-}$ & .001 & 0.01 & 0.99 \\
\hline Depression & $.531 * *_{-}$ & .001 & $.552 * *_{-}$ & .001 & 0.20 & 0.84 \\
\hline Anxiety & $.666 * *_{-}$ & .001 & $.540 * *_{-}$ & .001 & 1.28 & 0.10 \\
\hline
\end{tabular}

It is clear from Table (6) that the correlation between quality of life and pain level in the male sample was $(0.445)$ at a level of significance of (0.05) or less, while the correlation coefficient between quality of life and the level of pain in the female sample was (0.445) at the level of significance (0.05) or less, and that the value of $(\mathrm{Z})$ for the difference between them was $(0.01)$ at the level of (0.99) indicating that there is no difference between males and females in the correlation between quality of life and level of pain.

It was also found that the correlation coefficient between the quality of life and the level of depression in the male sample was (-0.531) with a level of significance of (0.05) or less, while the correlation between quality of life and the level of depression in the female sample was (-0.552) with a level of significance of (0.05) or less, and the value of $(\mathrm{Z})$ for the difference between them reached $(-0.20)$ at the level of $(0.84)$ indicating that there is no difference between males and females in the correlation between quality of life and level of depression. The correlation between quality of life and the level of anxiety in the male sample was (-0.66) with a level of significance of (0.05) or less, while the correlation between quality of life and the level of anxiety in the sample of females reached (0.54) with a level of significance of $(0.05)$ or less, and that the value of $(\mathrm{Z})$ for the difference between them reached (1.28) at level $(0.10)$ indicating that there is no difference between males and females in the correlation between quality of life and the level of anxiety.

Results related to the sixth question: Does the relationship between the quality of healthy life and the other variables (pain, anxiety, and depression) vary according to the level of education?

Table (7): Correlation between quality of life, level of pain, anxiety and depression according to the educational level variable

\begin{tabular}{lllllll}
\hline variable & \multicolumn{2}{l}{ Quality of life } & G & \multirow{2}{*}{ Significance } \\
\cline { 2 - 5 } & \multicolumn{2}{l}{ Secondary or less $\mathrm{n}=(81)$} & \multicolumn{2}{l}{ Academic N=117 } & & \\
& Link & Significance & Link & Significance & & \\
Pain & $-.377 * *_{1}$ & .001 & $.462 * *_{-}$ & .001 & -0.67 & 0.50 \\
Depression & $.523 * *_{-}$ & .001 & $.542 * *_{-}$ & .001 & -0.18 & 0.85 \\
Anxiety & $.590 * *_{-}$ & .001 & $.624 * *_{-}$ & .001 & -0.31 & 0.75 \\
\hline
\end{tabular}

It is clear from Table (7) that the correlation coefficient between quality of life and level of pain in a sample of secondary education was less than (-0.377) with a level of significance of $(0.05)$ or less. While the correlation between quality of life and the level of pain in a sample of university education was (-0.462) with a level of significance of $(0.05)$ or less, and that the value of $(Z)$ for the difference between them reached $(-0.67)$ at level $(0.50)$, indicating that there is no difference between those with secondary education and less and those with university education in the correlation between quality of life and level of pain.

It was also found that the coefficient of correlation between the quality of life and the level of depression in a sample of secondary education was less (-0.523) at a level of significance of (0.05) and less, while the correlation between quality 
of life and level of depression in a sample of university education was (0.542) at a level of significance of (0.05) and less, and the value of $(\mathrm{Z})$ of the difference between them reached (-0.18) at the level of 0.84 indicating that there is no difference between those with lower secondary education and those with university education in the correlation between quality of life and level of depression. The results showed that the correlation coefficient between the quality of life and the level of anxiety among a sample of those with secondary education and less was (-0.59) at a level of significance of (0.05) and less while the correlation between quality of life and the level of anxiety in a sample of university education was (-0.624) at a level of significance of $(0.05)$ and less, and that the value of $(\mathrm{Z})$ for the difference between them reached (-0.31) at the level of (0.75) indicating that there is no difference between those with secondary education or less and those with university education in the correlation between the quality of life and the level of anxiety.

Results related to the seventh question: To what extent does the level of pain, anxiety, and depression explain the quality of healthy life in patients with chronic low back pain? To answer this question, the multiple regression analysis, whose results are shown in Table 8, Shows how well the level of pain, anxiety, and depression explains the quality of healthy life among patients with chronic low back pain.

Table (8): Results of the multiple regression analysis of the explanatory power of each of the variables of pain, anxiety, and depression of quality of healthy life among patients with chronic low back pain

\begin{tabular}{lllllll}
\hline variable & Link & $\begin{array}{l}\text { The coefficient } \\
\text { of } \\
\text { determination }\end{array}$ & $\begin{array}{l}\text { Change in the } \\
\text { coefficient } \\
\text { determination }\end{array}$ & $\begin{array}{l}\text { So } \\
\text { of }\end{array}$ & & \\
\hline Anxiety & $0.545^{\mathrm{a}}$ & .297 & .297 & 84.667 & 0.001 \\
Level of pain & $0.642^{\mathrm{b}}$ & .412 & .115 & 38.828 & 0.001 \\
Depression & $0.676^{\mathrm{c}}$ & .457 & .045 & 16.359 & 0.001 \\
\hline
\end{tabular}

Table (8) shows the explanatory power factor of the concern variable of quality of life totaling (29.7\%), which is significant at (0.05) level and less, and that the level of pain has been interpreted individually (11.5\%) of the total variation in quality of life, and that the level of depression has accounted for (4.5\%) of the total variance in quality of life and that the sum explained by the variables (anxiety, pain level, and depression) totaling $45.7 \%$ of the total variation in quality of life.

Results related to the eighth question: Is there a relationship between the level of pain and the level of depression? To answer this question, the Pearson correlation coefficient was calculated between the level of pain and the level of depression and table (9) shows the results.

Table 9: Pearson correlation coefficient between pain level and depression level

\begin{tabular}{lll}
\hline \multicolumn{3}{l}{ Level of pain } \\
\hline & Correlation & Significance \\
Depression & 0.183 & 0.01 \\
\hline
\end{tabular}

From Table (9), the relationship between the level of pain and the level of depression was (0.183) at a mean level of $(0.05)$ and less, so there is a positive relationship between the level of pain and the level of depression.

Results related to the ninth question: Is there a relationship between the level of pain and the level of anxiety?

To answer this question Pearson correlation coefficient was calculated between pain level and anxiety level and Table (10) shows the results.

Table (10): Pearson correlation coefficient between pain level and anxiety level

\begin{tabular}{lll}
\hline \multicolumn{3}{c}{ Level of pain } \\
\hline & Correlation & Significance \\
Anxiety & 0.163 & 0.02 \\
\hline
\end{tabular}

In Table 10, the relationship between pain level and anxiety level was 0.163 at a mean of 0.05 and less, so there is a positive correlation between pain level and anxiety level.

\section{Discussion of the Results}

Clinical and psychological research tends to give greater self-expression to patients about the level of their health in many domains, where many researchers have studied the quality of life for patients suffering from chronic diseases and pain, including chronic low back pain. Many studies have indicated that there is a decline in the quality of life in people with chronic low back pain. 
Studies have also examined the psychological factors associated with chronic low back pain. Several studies have concluded that there are interrelationships between chronic pain and psychological factors (anxiety, depression, stress), and others that have correlations between psychological factors and chronicity, there are several studies that confirm the quality of the correlation between depression and disability in patients with low back pain. The study of Jarrar went even further considering that the rate of interpretation of disability through depression is higher than that of the pain itself. But little is known about the relationship between the variables (pain, anxiety, and depression) and quality of life in patients with chronic low back pain. specially that the quality of healthy life scale used in the current study has two main aspects: the first is mental health, the second is physical health, and therefore the findings of the present study are not only new but also they are rare; but found clear and strong links and relationships between pain and anxiety as well as between pain and depression on the one hand and between pain, anxiety and depression on the other. The results of this study also showed the extent to which the variables (pain, anxiety, and depression) contributed to the interpretation of the quality of life of patients with chronic low back pain. Biological, psychological and social factors affect perception of pain. Chronic pain may be caused by the interaction of these factors, and even these factors contribute to the interpretation of various mental disorders such as anxiety and depression, this is consistent with the psychosocial model of psychological and physical disorders, especially for chronic low back pain patients. There is a broad appreciation and great importance for the formulation of these dimensions to be explained by the psychosocial biological model of these patients (Waddell G, 2004). As well as the quality of healthy life, these psychological, social and biological dimensions are present and represented in the items of (SF-36) scale used in the current study, thus making it more accessible to physicians and physiotherapists to understand how patients perceive their health and quality of life, thus providing indicators of the impact of quality of healthy life in the sub-domains of the scale.

\section{Recommendations}

The researchers set some recommendations

1. To prepare a multidisciplinary team in hospitals, medical centers, physiotherapy centers and specialized rehabilitation centers to evaluate and provide medical and psychological care to patients suffering from chronic pain.

2. Conducting further studies on psychological factors related to chronic pain including anxiety trait.

3. To adopt the course of clinical health psychology as a compulsory subject within the academic courses in various medical studies to achieve a higher understanding of what the pain and its aspects within the bio-psychological biological model.

4. Expanding the work of psychological intervention services for patients with chronic low back pain and patients complaining of chronic pain in order to provide appropriate treatment for patients.

5. Conducting studies on integrated psychological therapy programs with doctors and physiotherapists in order to achieve the desired priority in the treatment of patients with chronic low back pain and related psychological symptoms, especially anxiety.

6. Adopting a quality of healthy life scale to assess the effectiveness of different treatment programs in chronic pain.

\section{References}

Adorno ML, Brasil-Neto JP. (2013). Assessment of the quality of life through the SF-36 questionnaire in patients with chronic nonspecific low back pain. Acta Ortop Bras. Jul; 21(4):202-7

Al-Dawood, A. (2007) Quality of life among Saudi diabetic patients, Thesis (M. Sc. in Nursing), Jordan University of Science and Technology

Al-Hourani, M. (2007) Quality of life of northern Jordanians using SF-36 .Thesis (M. Sc. in Public Health)--Jordan University

Alshami, A. (2014) Physical and psychological aspects of low back pain among Saudi patients: A case-control study. Saudi J Med Med Sci; 2, pp.24-9

Baena-Beato PÁ, Artero EG, Arroyo-Morales M, Robles-Fuentes A, Gatto-Cardia MC, Delgado-Fernández M. (2014). Aquatic therapy improves pain, disability, quality of life, body composition and fitness in sedentary adults with chronic low back pain, A controlled clinical trial. Clin Rehabil; 28(4):350-60

Berg-Emons, R., Schasfoort, F., Vos, L., Bussmann, J., \& Stam, H. (2007). Impact of chronic pain on everyday physical activity, European Journal Of Pain, 11(5), 587-593. doi:10.1016/j.ejpain.2006.09.003

Hong, J. H., Kim, H. D., Shin, H. H., \& Huh, B. (2014) Assessment of depression, anxiety, sleep disturbance, and quality of life in patients with chronic low back pain in Korea. Korean Journal of Anesthesiology, 66(6), 444450. doi:10.4097/kjae.2014.66.6.444 
Jarrar, R. (2011) levels of pain and depression and their relationship to feeling helpless in a sample of low back pain patients in Amman, unpublished Master Thesis. University of Jordan, Amman, Jordan

Lindsay, P. (2000). Reference in clinical psychology for adults. (Translated by Faraj, Safwat). Cairo: Anglo Egyptian

Perquin CW, Hazebroek-Kampschreur AA, Hunfeld JA, Bohnen AM, van Suijlekom-Smit LW, Passchier J, van der Wouden JC. Pain in children and adolescents: a common experience. Pain. 2000 Jul;87(1):51-8. PubMed PMID: 10863045.

Pizzo PA, Clark NM.(2012), Alleviating suffering 101—pain relief in the United States.NEngl J Med;366(3):197-199

Sirianni J, Ibrahim M, Patwardhan A (2015). Chronic pain syndromes, mechanisms, and current treatments.Prog Mol Biol Transl Sci.; 131:565-611

Spielberger CD, Gorsuch RL, Lushene R, Vagg PR, Jacobs GA.(1983) Manual for the State-Trait Anxiety Inventory for Adults. Palo Alto, CA: Mind Garden, Inc; 1983

Tsang, A., Von Korff, M., Lee, S., Alonso, J., Karam, E., \& Angermeyer, M. et al. (2008). Common Chronic Pain Conditions in Developed and Developing Countries: Gender and Age Differences and Comorbidity With Depression-Anxiety Disorders. The Journal Of Pain, 9(10), 883-891. doi:10.1016/j.jpain.2008.05.005

Waddell G.( 2004) The back pain revolution. 2nd ed. Churchill Livingstone

WHO (1946), Preamble to the Constitution of the World Health Organization. WHO, New York, USA 